\title{
Heterosis effect of some onion (Allium cepa L.) characteristics
}

\section{RÖH W. DORUCHOWSKI}

Department of Vegetable Breeding and Genetics, Research Institute of Vegetable Crops, ul. 22 Lipca 1/3, 96-100 Skierniewice, Poland

(Received: April 2, 1986)

\begin{abstract}
The object of the studies in 1977 was a population of $15 \mathrm{~F}_{1}$ hybrids, $11 \mathrm{~F}_{2}$ progenies, 16 parental forms ( 8 malesterile $\mathrm{A}$ lines and 3 inbred $\mathrm{C}$ lines). Heterosis of onion bulb weight, height, diameter was high. The heterosis of bulb firmness, skin adherence and sugar content was not high. The heterosis of other characteristics was rather low.
\end{abstract}

\section{INTRODUCTION}

Development of $F_{1}$ hybrids with bulbs possessing desirable utilitarian traits is possible due to studies on the crossing ability of maternal, that is, male-sterile, lines and inbred paternal lines, C, having fertile pollen ( $\mathrm{J}$ ones and Clarke, 1943-1947; J ones and Davis, 1944; Erickson and Gabelman, 1954; Kobabe, 1958; Doruchowski, 1968, 1969, 1971, 1978; Brezhniev, 1971; Dowker and Fenne 11, 1974; Feltz, 1975; Arunachalm, 1977).

Parental lines are usually developed from varieties having the most desirable traits. The crossing ability of a certain number of hybrids and their production value, mainly in respect to yield and uniformity of bulb, characteristics, were studied in 1966-1967 (D o r u ch ow ski, 1968). In this study, the results are presented on the heterosis of the following traits: bulb weight, height, diameter, collar thickness, dry skin thickness, bulb firmness, root disc diameter and dry matter and sugar contents.

\section{MATERIALS AND METHODS}

The studies were done at the Research Institute of Vegetable Crops in 1977-1978. The materials used in this study are described in the first part of this series of papers (Dor uchowski, 1986). 
Bulb weight is expressed in dag, bulb height, diameter, collar thickness and root disc diameter in $\mathrm{cm}$, dry skin thickness in $\mu$, and bulb firmness in readings from 1-100 of a Type No. 2 durometer from the Shore Instrument and MFG Co., Inc. 90-35 Van Wyck Expressway, Jamaica, N.Y. 11435 U.S.A. Samples numbering from 19-100 bulbs were picked randomly from three replications.

The chemical composition of the onions determined in cooperation with the Departmen of Food Processing and Freezing of the Research Institute of Vegetable Crops in Skierniewice. The dry matter content in percent of fresh weight was determined by initial drying at a temperature of $60-70^{\circ} \mathrm{C}$, next at $105^{\circ} \mathrm{C}$ until a constant weight was attained. Additionally, dry matter was determined refractometrically.

The simple and total sugar contents were determined by the Loof-Schoorl method in percentage of fresh weight. The differences between the mean values for the parental forms and their $F_{1}$ and $F_{2}$ hybrids were evaluated using Student's $t$ test at a significance level of $\alpha=0.05$.

The heterosis effect (Tables 1-8) was expressed as the percentage ratio of the average values for $F_{1}$ hybrids to the mean for the parental line with the higher trait value:

$$
\mathbf{H}=\overline{\mathrm{X}}_{\mathrm{F}_{1}}: \overline{\mathrm{Xp}}_{\max } \cdot 100 \%
$$

\section{RESULTS}

The average onion bulb weight in most of the maternal components, that is, male-sterile $\mathrm{A}$ lines, was higher than in the paternal, inbred $\mathrm{C}$ lines, with the exception of A-63, A-16, C-177b, C-181 and C-16 (Table 1). The bulb weight of $8 F_{1}$ hybrids among the 15 in 1977, and $4 F_{1}$ hybrids among 12 in 1978, was significantly higher than that of both parental components. The best in this respect were, in $1977-F_{1}$ hybrids: $A-16 \times C-16$ and $A-19 \times C-19$, which exhibited the highest heterosis effect. The weight of $3 \mathrm{~F}_{1}$ hybrids was on the same level as that of line $\mathrm{A}$, of 3 others, below it. Only $\mathrm{F}_{2}$ hybrid A-16 $\times$ C-141 had an average weight somewhat smaller than $F_{1}$. The remaining hybrids had weights significantly smaller than the weight of $F_{1}$ hybrid bulbs and similar to that of one of the parents, due to segregation of traits.

The very unfavorable atmospheric conditions for onion growth which occurred in 1978, negatively influenced heterosis, which was slightly noticeable in the weight of $F_{1}$ hybrid bulbs. In connection with this, the mean bulb, weight for both parental and $F_{1}$ hybrid forms was very differentiated and, in most cases, low. 
Table 1

Mean values $(\vec{x})$ and heterosis effect of onion bulb weight (dag)

\begin{tabular}{|c|c|c|c|c|c|c|c|c|c|c|c|}
\hline \multirow{3}{*}{ No. } & \multirow{3}{*}{\multicolumn{2}{|c|}{ Hybrid formula }} & \multicolumn{5}{|c|}{1977} & \multicolumn{4}{|c|}{1978} \\
\hline & & & \multirow[b]{2}{*}{ line $A$} & \multicolumn{2}{|c|}{$\bar{x}$} & \multicolumn{2}{|r|}{$\begin{array}{c}\text { heterosis } \\
\text { efect }\end{array}$} & \multicolumn{3}{|c|}{$\bar{x}$} & \multirow{2}{*}{$\begin{array}{c}\begin{array}{c}\text { heterosis } \\
\text { effect }\end{array} \\
\begin{array}{c}\mathrm{H} \text { according } \\
\text { to } P_{\max }\end{array}\end{array}$} \\
\hline & & & & line $C$ & $F_{1}$ & $F_{2}$ & $\begin{array}{c}\mathbf{H} \text { according } \\
\text { to } \mathrm{P}_{\max }\end{array}$ & line $A$ & line $C$ & $F_{1}$ & \\
\hline 1. & A- 6 & $\times C-177 a$ & $11.9 \mathrm{c}$ & $9.4 b$ & $11.6 \mathrm{c}$ & $7.6 \mathrm{a}$ & 97.5 & $\ldots$ & - & - & - \\
\hline 2. & A-16 & $\times \mathrm{C}-177 \mathrm{a}$ & $11.0 \mathrm{c}$ & $9.4 b$ & $15.7 \mathrm{~d}$ & $8.5 a$ & 142.7 & $13.0 \mathrm{~b}$ & $6.6 a$ & $12.0 \mathrm{~b}$ & 92.3 \\
\hline 3. & A-54 & $\times \mathrm{C}-177 \mathrm{a}$ & $16.7 \mathrm{~d}$ & $9.4 b$ & $14.1 \mathrm{c}$ & $6.0 \mathrm{a}$ & 84,4 & $9.5 b$ & $6.6 a$ & $11.8 \mathrm{c}$ & 124.2 \\
\hline 4. & A-58 & $\times \mathrm{C}-177 \mathrm{a}$ & $13.4 b$ & $9.4 \mathrm{a}$ & $10.3 \mathrm{a}$ & - & 76.9 & - & - & - & - \\
\hline 5. & A-125 & $\times C-177 a$ & $12.1 b$ & $9.4 \mathrm{a}$ & $14.4 \mathrm{c}$ & $8.6 a$ & 119.0 & $7.5 \mathrm{a}$ & $6.6 \mathrm{a}$ & $7.8 \mathrm{a}$ & 104.0 \\
\hline 6. & A-19 & $\times \mathrm{C}-177 \mathrm{a}$ & $11.9 \mathrm{c}$ & $9.4 \mathrm{a}$ & $13.8 \mathrm{c}$ & - & 116.0 & - & - & 一 & - \\
\hline 7. & A-181 & $\times C-177 a$ & $18.1 \mathrm{c}$ & $9.4 \mathrm{a}$ & $14.0 \mathrm{~b}$ & - & 77.3 & $11.1 \mathrm{c}$ & $6.6 \mathrm{a}$ & $8.7 \mathrm{~b}$ & 78.4 \\
\hline 8. & A- 63 & $\times C-177 b$ & $9.2 \mathrm{a}$ & $11.3 \mathrm{~b}$ & $13.1 \mathrm{c}$ & $10.6 b$ & 115.9 & - & - & - & - \\
\hline 9. & A-16 & $\times \mathrm{C}-141$ & $11.0 \mathrm{~b}$ & $9.4 \mathrm{a}$ & $13.7 \mathrm{c}$ & $12.5 \mathrm{c}$ & 124.5 & - & 一 & - & - \\
\hline 10. & A-58 & $\times$ C-141 & $13.4 b$ & $9.4 \mathrm{a}$ & $13.3 b$ & - & 99.2 & $10.4 \mathrm{c}$ & $5.8 \mathrm{a}$ & $8.0 \mathrm{~b}$ & 76.9 \\
\hline 11. & A-12 & $\times$ C-141 & - & - & - & - & - & $9.4 b$ & $5.8 \mathrm{a}$ & $12.8 \mathrm{c}$ & 136.2 \\
\hline 12. & A- 125 & $\times$ C-181 & $12.1 \mathrm{~b}$ & $15.0 \mathrm{c}$ & $16.9 \mathrm{~d}$ & $8.1 \mathrm{a}$ & 112.7 & $7.5 \mathrm{a}$ & $9.1 \mathrm{~b}$ & $14.2 \mathrm{c}$ & 156.0 \\
\hline 13. & A-181 & $\times$ C-181 & - & - & - & - & - & $11.1 \mathrm{~b}$ & $9.1 \mathrm{a}$ & $13.0 \mathrm{c}$ & 117.1 \\
\hline 14. & A-16 & $\times C-16$ & $11.0 \mathrm{a}$ & $15.5 \mathrm{c}$ & $15.7 \mathrm{c}$ & $13.7 \mathrm{~b}$ & 101.3 & $13.0 \mathrm{~b}$ & $11.4 \mathrm{~b}$ & $9.5 \mathrm{a}$ & 73.1 \\
\hline 15. & A-6 & $\times$ C -6 & $11.9 b c$ & $7.5 a$ & $12.8 \mathrm{c}$ & $11.2 \mathrm{~b}$ & 107.6 & - & - & - & - \\
\hline 16. & A- 125 & $\times$ C-199 & $12.1 \mathrm{~b}$ & $10.2 \mathrm{a}$ & $14.2 \mathrm{c}$ & $11.1 \mathrm{ab}$ & 117.3 & - & - & - & - \\
\hline 17. & A-19 & × C-19 & $11.9 \mathrm{a}$ & $11.0 \mathrm{a}$ & $17.3 \mathrm{c}$ & $13.8 \mathrm{~b}$ & 145.4 & - & - & - & - \\
\hline 18. & A-16 & $\times$ C -9 & - & - & - & - & - & $13.0 \mathrm{~b}$ & $6.4 a$ & $11.9 \mathrm{~b}$ & 91.5 \\
\hline 19. & A-101 & $\times \mathrm{C}-9$ & - & - & - & - & - & $11.2 \mathrm{~b}$ & $6.4 a$ & $10.6 b$ & 94.6 \\
\hline 20. & A-1 81 & $\times$ C -9 & - & - & - & - & - & $11.1 \mathrm{~b}$ & $6.4 \mathrm{a}$ & $15.5 \mathrm{c}$ & 139.6 \\
\hline
\end{tabular}

Mean values denoted by the same tetter do not differ significantly $(a \neq 0.05)$ by Student's. $t$ - test. Evaluation of differences was done within the hybrid formula. 
The mean bulb height was, in most of the paternal components, significantly higher than in the maternal ones, and also in some cases, greater than in $F_{1}$ and $F_{2}$ hybrids (Table 2). The onion bulbs in the paternal lines $\mathrm{C}$ had a more or less elongated shape and so their height was greater than their diameter. The height of $F_{1}$ hybrid bulbs was intermediate in comparison with parental forms, or significantly higher in $\mathrm{F}_{1}$ hybrids $(\mathrm{A}-16 \times \mathrm{C}-177 \mathrm{a}),(\mathrm{A}-19 \times \mathrm{C}-177 \mathrm{a}),(\mathrm{A}-6 \times \mathrm{C}-6)$ and $(\mathrm{A}-125 \times$ $\mathrm{C}-199)$ than in the higher of the parents, exhibiting evident heterosis. In $F_{2}$, this trait was close to parental from values because of trait segregation.

The bulb diameter in most A lines was significantly greater than in $\mathrm{C}$ lines and in some of the $\mathrm{F}_{1}$ hybrids (Table 3 ). However, the average diameter of bulbs from $C$ lines was significantly smaller than in $F_{1}$ hybrids. The $F_{1}$ hybrids which had a large mean bulb diameter also had a large mean weight and exhibited the highest heterosis of both diameter and weight: $F_{1}(A-16 \times C-177 a),(A-16 \times C-141),(A-6 \times C-6)$ and $(A-19 \times$ $\mathrm{C}-19)$. Due to this, there is a realistic possibility of increasing the yield by introducing these $\mathrm{F}_{1}$ hybrids into production.

The mean bulb diameter of $F_{2}$ hybrids was similar to that in lines $A$ and $C$ because of segregation of these traits and parental characteristics thereby becoming evident.

The mean collar thickness was only sligthly higher $(5 \%)$ in $3 \mathrm{~F}_{1}$ hybrids (A-63 $\times$ C-177b), (A-6 $\times$ C-6) and (A-125 $\times$ C-199) showing heterosis (Table 4). As results from this, most of the hybrids did not have thick collars, which is a very desirable trait from the standpoint of production.

All of the $\mathrm{C}$ lines, with the exception of C-19, had a mean dry skin thickness which was larger than that of A lines (Table 5), Heterosis of dry skin thickness was not found in the majority of $F_{1}$ hybrids, with the exception of $(\mathrm{A}-125 \times \mathrm{C}-181)$. The results suggest that in order to improve the quality of the dry skin in $F_{1}$ hybrids, the parental components should be chosen so that they both have thick, strong and well adhering dry skin. Individuals of better quality than that of A line can be selected from $\mathrm{F}_{2}$ hybrids.

The mean bulb firmness in 1977 in $2 F_{1}$ hybrids was significantly greater than in line $\mathrm{C}$ in comparison with bulb firmness in line $\mathrm{A}$ and the remaining $F_{1}$ and $F_{2}$ hybrids, with the exception of line A-63 (Table 6). Evident heterosis was found only in $F_{1}(A-19 \times C-19)$. The bulb firmness in $F_{2}$ hybrids was significantly lower than in $F_{1}$ hybrids. The $F_{2}$ generation $(\mathrm{A}-16 \times \mathrm{C}-141)$ was an exception, having a higher value. The differences were significant at the $\alpha=0.05$ level.

In order to improve bulb firmness in $F_{1}$ hybrids, a large number of various parental forms or lines with firm bulbs are necessary. These 
Table 2

Mean values $(\bar{x})$ and heterosis effect of onion bulb height $(\mathrm{cm})$

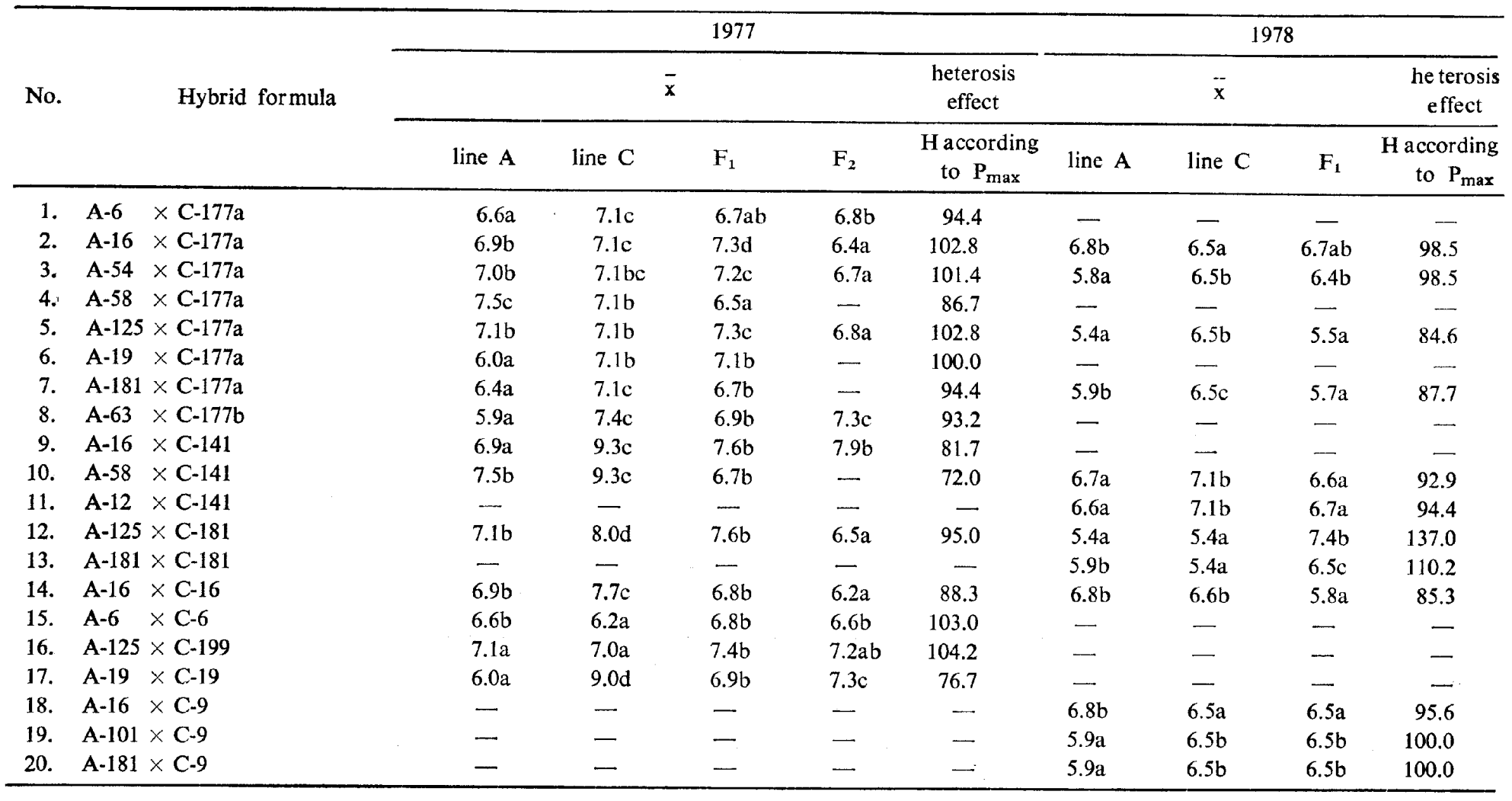

Explanations as in Table 1. 
T a ble 3

Mean values $(\bar{x})$ and heterosis effect of onion bulb diameter $(\mathrm{cm})$

\begin{tabular}{|c|c|c|c|c|c|c|c|c|c|c|c|}
\hline \multirow{3}{*}{ No. } & & \multirow{3}{*}{ Hybrid formula } & \multicolumn{5}{|c|}{1977} & \multicolumn{4}{|c|}{1978} \\
\hline & & & \multicolumn{4}{|c|}{$\bar{x}$} & \multirow{2}{*}{$\begin{array}{l}\begin{array}{c}\text { heterosis } \\
\text { effect }\end{array} \\
\begin{array}{c}\text { H according } \\
\text { to } P_{\max }\end{array}\end{array}$} & \multicolumn{3}{|c|}{$\bar{x}$} & \multirow{2}{*}{$\begin{array}{c}\begin{array}{c}\text { hetcrosis } \\
\text { effect }\end{array} \\
\begin{array}{c}\text { H according } \\
\text { to } P_{\max }\end{array}\end{array}$} \\
\hline & & & line $\mathbf{A}$ & line $C$ & $F_{1}$ & $F_{2}$ & & line $A$ & line $C$ & $F_{1}$ & \\
\hline 1. & A-6 & $\times$ C-177a & $6.4 \mathrm{c}$ & $5.5 b$ & $6.3 \mathrm{c}$ & $5.1 \mathrm{a}$ & 98.4 & - & - & - & - \\
\hline 2. & A-16 & $\times C-177 a$ & $6.2 b$ & $5.5 \mathrm{a}$ & $7.0 \mathrm{c}$ & $5.5 \mathrm{a}$ & 112.9 & $6.6 \mathrm{c}$ & $4.7 \mathrm{a}$ & $6.4 \mathrm{~b}$ & 97.0 \\
\hline 3. & A-54 & $\times C-177 a$ & $7.4 \mathrm{~d}$ & $5.5 \mathrm{~b}$ & $6.7 \mathrm{c}$ & $4.7 \mathrm{a}$ & 90.5 & $6.0 \mathrm{~b}$ & $4.7 \mathrm{a}$ & $6.4 c$ & 106.7 \\
\hline 4. & A- 58 & $\times C-177 a$ & $6.7 \mathrm{c}$ & $5.5 \mathrm{a}$ & $6.1 \mathrm{~b}$ & - & 91.0 & - & - & - & - \\
\hline 5. & A-125 & $\times \mathrm{C}-177 \mathrm{a}$ & $6.4 \mathrm{~b}$ & $5.5 \mathrm{a}$ & $6.7 \mathrm{c}$ & $5.3 \mathrm{a}$ & 104.7 & $5.3 \mathrm{~b}$ & $4.7 \mathrm{a}$ & $5.0 \mathrm{ab}$ & 94.3 \\
\hline 6. & A-19 & $\times \mathrm{C}-177 \mathrm{a}$ & $6.8 \mathrm{~b}$ & $5.5 \mathrm{a}$ & $6.6 \mathrm{~b}$ & - & 97.1 & - & - & - & - \\
\hline 7. & A-181 & $\times \mathrm{C}-177 \mathrm{a}$ & $7.8 \mathrm{c}$ & $5.5 \mathrm{a}$ & $6.9 \mathrm{~b}$ & - & 88.5 & $6.3 \mathrm{c}$ & $4.7 \mathrm{a}$ & $5.8 \mathrm{~b}$ & 92.1 \\
\hline 8. & A-63 & $\times C-177 b$ & $5.8 \mathbf{a}$ & $5.9 \mathrm{a}$ & $6.6 b$ & $5.8 \mathrm{a}$ & 111.9 & - & - & - & - \\
\hline 9. & A-16 & $\times C-141$ & $6.2 b$ & $4.7 \mathrm{a}$ & $6.5 \mathrm{c}$ & $6.1 \mathrm{~b}$ & 104.8 & - & - & - & - \\
\hline 10. & A-58 & $\times \mathrm{C}-141$ & $6.7 \mathrm{~b}$ & $4.7 \mathrm{a}$ & $6.5 b$ & - & 97.0 & $6.1 \mathrm{c}$ & $4.2 \mathrm{a}$ & $5.3 \mathrm{~b}$ & 86.9 \\
\hline 11. & $A-12$ & $\times C-141$ & - & - & - & - & - & $5.5 b$ & $4.2 \mathrm{a}$ & $6.5 c$ & 118.2 \\
\hline 12. & A-125 & $\times$ C- 181 & $6.4 b$ & $6.5 b$ & $6.9 \mathrm{c}$ & $5.3 a$ & 106.1 & $5.3 \mathrm{a}$ & $5.7 b$ & $6.4 \mathrm{c}$ & 112.3 \\
\hline 13. & A-181 & $\times C-181$ & - & - & - & - & - & $6.3 b$ & $5.7 \mathrm{~b}$ & $6.5 b$ & 103.2 \\
\hline 14. & A-16 & $\times \mathrm{C}-16$ & $6.2 \mathrm{a}$ & $6.8 \mathrm{~b}$ & $7.1 \mathrm{c}$ & $7.0 \mathrm{bc}$ & 104.4 & $6.6 \mathrm{~b}$ & $6.1 \mathrm{a}$ & $5.9 \mathrm{a}$ & 89.4 \\
\hline 15. & A-6 & $\times C-6$ & $6.4 b c$ & $5.5 \mathrm{a}$ & $6.5 c$ & $6.2 \mathrm{~b}$ & 101.6 & - & - & - & - \\
\hline 16. & A-125 & $\times \mathrm{C}-199$ & $6.4 b$ & $5.7 \mathrm{a}$ & $6.6 b$ & $5.8 \mathrm{a}$ & 103.1 & - & - & - & - \\
\hline 17. & A-19 & $\times$ C-19 & $6.8 \mathrm{~b}$ & $5.5 \mathrm{a}$ & $7.3 \mathrm{c}$ & $6.7 \mathrm{~b}$ & 107.3 & - & - & - & - \\
\hline 18. & A-16 & $\times C-9$ & - & - & - & - & - & $6.6 b$ & $4.6 a$ & $6.4 b$ & 97.0 \\
\hline 19. & A-101 & $\times$ C -9 & - & - & - & - & - & $6.3 b$ & $4.6 \mathrm{a}$ & $6.0 \mathrm{~b}$ & 95.2 \\
\hline 20. & A-181 & $\times$ C -9 & - & - & - & - & - & $6.3 b$ & $4.6 \mathrm{a}$ & $7.1 \mathrm{c}$ & 112.7 \\
\hline
\end{tabular}

Explanations as in Table 1. 
Table 4

Mean values $(\bar{x})$ and heterosis effect of collar thickness $(\mathrm{cm})$

\begin{tabular}{|c|c|c|c|c|c|c|c|c|c|c|c|}
\hline \multirow{3}{*}{ No. } & & \multirow{3}{*}{ Hybrid formula } & \multicolumn{5}{|c|}{1977} & \multicolumn{4}{|c|}{1978} \\
\hline & & & \multicolumn{4}{|c|}{$\bar{x}$} & \multirow{2}{*}{$\begin{array}{c}\text { heterosis } \\
\text { effect }\end{array}$} & \multicolumn{3}{|c|}{$\bar{x}$} & \multirow{2}{*}{$\begin{array}{c}\begin{array}{c}\text { heterosis } \\
\text { effect }\end{array} \\
\mathrm{H} \text { according } \\
\text { to } \mathrm{P}_{\max }\end{array}$} \\
\hline & & & line $\mathrm{A}$ & line $\mathrm{C}$ & $F_{1}$ & $\mathrm{~F}_{2}$ & & line $A$ & line $C$ & $F_{1}$ & \\
\hline 1. & A-6 & $\times \mathrm{C}-177 \mathrm{a}$ & $1.8 \mathrm{c}$ & $1.8 \mathrm{c}$ & $1.7 \mathrm{~b}$ & $1.2 \mathrm{a}$ & 94.4 & - & - & - & - \\
\hline 2. & A-16 & $\times C-177 a$ & $1.7 \mathrm{~b}$ & $1.8 \mathrm{c}$ & $1.8 \mathrm{c}$ & $1.3 \mathrm{a}$ & 100.0 & $1.5 \mathrm{a}$ & $1.5 \mathrm{a}$ & $1.5 \mathrm{a}$ & 100.0 \\
\hline 3. & A-54 & $\times C-177 a$ & $1.9 \mathrm{c}$ & $1.8 \mathrm{~b}$ & $1.9 \mathrm{c}$ & $1.1 \mathrm{a}$ & 100.0 & $1.4 \mathrm{a}$ & $1.5 b$ & $1.4 \mathrm{a}$ & 93.3 \\
\hline 4. & A-58 & $\times C-177 a$ & $1.8 \mathrm{~b}$ & $1.8 \mathrm{~b}$ & $1.6 \mathrm{a}$ & - & 88.9 & - & - & - & - \\
\hline 5. & A- 125 & $\times C-177 a$ & $1.8 \mathrm{~b}$ & $1.8 \mathrm{~b}$ & $2.0 \mathrm{c}$ & $1.3 \mathrm{a}$ & 111.1 & $1.2 \mathrm{a}$ & $1.5 \mathrm{~b}$ & $1.3 \mathrm{ab}$ & 86.7 \\
\hline 6. & A-19 & $\times C-177 a$ & $1.4 \mathrm{a}$ & $1.8 \mathrm{~b}$ & $1.8 \mathrm{~b}$ & - & 100.0 & - & - & - & - \\
\hline 7. & A-181 & $\times \mathrm{C}-177 \mathrm{a}$ & $1.6 \mathrm{a}$ & $1.8 \mathrm{c}$ & $1.7 \mathrm{~b}$ & - & 94.4 & $1.5 \mathrm{~b}$ & $1.5 \mathrm{~b}$ & $1.4 \mathrm{a}$ & 93.3 \\
\hline 8. & A -63 & $\times C-177 b$ & $1.9 b$ & $1.9 \mathrm{~b}$ & $2.0 \mathrm{c}$ & $1.5 \mathrm{a}$ & 105.3 & - & - & - & $一$ \\
\hline 9. & A-16 & $\times C-141$ & $1.7 \mathrm{~b}$ & $1.8 \mathrm{c}$ & $1.8 \mathrm{c}$ & $1.4 \mathrm{a}$ & 100.0 & - & - & - & - \\
\hline 10. & A-58 & $\times C-141$ & $1.8 \mathrm{~b}$ & $1.8 \mathrm{~b}$ & $1.5 \mathrm{a}$ & - & 83.3 & $1.5 \mathrm{c}$ & $1.2 \mathrm{a}$ & $1.3 \mathrm{~b}$ & 86.7 \\
\hline 11. & A-12 & $\times C-141$ & - & - & - & - & - & $1.5 b$ & $1.2 \mathrm{a}$ & $1.5 b$ & 100.0 \\
\hline 12. & A-125 & $\times C-181$ & $1.8 \mathrm{c}$ & $1.9 \mathrm{c}$ & $1.4 \mathrm{~b}$ & $1.2 \mathrm{a}$ & 73.7 & $1.2 \mathrm{a}$ & $1.3 b$ & $1.5 \mathrm{c}$ & 115.4 \\
\hline 13. & A-181 & $\times C-181$ & - & - & - & - & $\ldots$ & $1.5 b$ & $1.3 \mathrm{a}$ & $1.5 b$ & 100.0 \\
\hline 14. & A-16 & $\times C-16$ & $1.7 \mathrm{c}$ & $1.8 \mathrm{~d}$ & $1.6 \mathrm{~b}$ & $1.2 \mathrm{a}$ & 88.9 & $1.5 \mathrm{~b}$ & $1.6 \mathrm{~b}$ & $1.3 \mathrm{a}$ & 81.2 \\
\hline 15. & A- 6 & $\times C-6$ & $1.8 \mathrm{c}$ & $1.6 \mathrm{~b}$ & $1.9 \mathrm{~d}$ & $1.4 \mathrm{a}$ & 105.5 & - & - & - & - \\
\hline 16. & A-125 & $\times$ C-199 & $1.8 \mathrm{~b}$ & $1.8 \mathrm{~b}$ & $1.9 \mathrm{c}$ & $1.4 \mathrm{a}$ & 105.5 & - & - & - & - \\
\hline 17. & A-19 & $\times$ C-19 & $1.4 \mathrm{a}$ & $1.9 \mathrm{~d}$ & $1.8 \mathrm{c}$ & $1.5 b$ & 94.7 & - & - & $\ldots$ & - \\
\hline 18. & A-16 & $\times C-9$ & $\ldots$ & - & - & - & - & $1.5 \mathrm{~b}$ & $1.3 \mathrm{a}$ & $1.5 b$ & 100.0 \\
\hline 19. & A-101 & $\times$ C-9 & - & - & - & - & - & $1.4 \mathrm{~b}$ & $1.3 \mathrm{a}$ & $1.5 \mathrm{~b}$ & 107.1 \\
\hline 20. & A-181 & $\times C-9$ & - & - & - & - & - & $1.5 \mathrm{c}$ & $1.3 \mathrm{a}$ & $1.4 \mathrm{~b}$ & 93.3 \\
\hline
\end{tabular}

Explanations as in Table 1. 
Table 5

Mean values $(\bar{x})$ and heterosis effect of dry skin thickness $(\mu)$

\begin{tabular}{|c|c|c|c|c|c|c|c|c|c|c|c|c|}
\hline \multirow{3}{*}{ No. } & & \multirow{3}{*}{ Hybrid formula } & & \multicolumn{5}{|c|}{1977} & \multicolumn{4}{|c|}{1978} \\
\hline & & & & \multicolumn{4}{|c|}{$\bar{x}$} & \multirow{2}{*}{$\begin{array}{c}\begin{array}{c}\text { heterosis } \\
\text { effect }\end{array} \\
\begin{array}{l}\text { according } \\
\text { to } \mathrm{P}_{\max }\end{array}\end{array}$} & \multicolumn{3}{|c|}{$\bar{x}$} & \multirow{2}{*}{$\begin{array}{c}\begin{array}{c}\text { heterosis } \\
\text { effect }\end{array} \\
\text { H according } \\
\text { to } P_{\max }\end{array}$} \\
\hline & & & & line $\mathbf{A}$ & line $C$ & $F_{1}$ & $\mathrm{~F}_{2}$ & & linea $\mathrm{A}$ & line $\mathrm{C}$ & $\mathrm{F}_{1}$ & \\
\hline 1. & A-6 & $\times \mathrm{C}-177 \mathrm{a}$ & & $0.2 \mathrm{a}$ & $0.6 \mathrm{c}$ & $0,5 b$ & $0.5 b$ & 83.3 & - & - & - & - \\
\hline 2. & A-16 & $\times C-177 a$ & & $0.3 \mathrm{a}$ & $0.6 \mathrm{c}$ & $0.4 \mathrm{~b}$ & $0.4 \mathrm{~b}$ & 66.7 & $0.3 \mathbf{a}$ & $0.6 b$ & $0.3 \mathrm{a}$ & 50.0 \\
\hline 3. & A-54 & $\times \mathrm{C}-177 \mathrm{a}$ & & $0.3 \mathrm{a}$ & $0.6 \mathrm{~d}$ & $0.4 b$ & $0.5 \mathrm{c}$ & 66.7 & $0.2 \mathrm{a}$ & $0.6 c$ & $0.3 \mathrm{~b}$ & 50.0 \\
\hline 4. & A-58 & $\times C-177 a$ & & $0.3 \mathrm{a}$ & $0.6 \mathrm{c}$ & $0.4 \mathrm{~b}$ & $\ldots$ & 66.7 & - & $\longrightarrow$ & $\longrightarrow$ & $\longrightarrow$ \\
\hline 5. & A-125 & $\times C-177 a$ & & $0.3 a$ & $0.6 \mathrm{c}$ & $0.3 a$ & $0.4 b$ & 50.0 & $0.3 \mathrm{a}$ & $0.6 c$ & $0.4 b$ & 66.7 \\
\hline 6. & A-19 & $\times \mathrm{C}-177 \mathrm{a}$ & & $0.5 \mathrm{a}$ & $0.6 \mathrm{~b}$ & $0.7 \mathrm{c}$ & $\longrightarrow$ & 116.7 & $\longrightarrow$ & - & - & - \\
\hline 7. & A-181 & $\times \mathrm{C}-177 \mathrm{a}$ & & $0.4 \mathrm{a}$ & $0.6 \mathrm{~b}$ & $0.6 \mathrm{~b}$ & - & 100.0 & $0.4 \mathrm{a}$ & $0.6 \mathrm{~b}$ & $0.6 b$ & 100.0 \\
\hline 8. & $A-63$ & $\times \mathrm{C}-177 \mathrm{a}$ & & $0.4 a$ & $0.5 b$ & $0.5 b$ & $0.4 \mathrm{a}$ & 100.0 & - & 一 & - & - \\
\hline 9. & A-16 & $\times C-141$ & & $0.3 \mathrm{a}$ & $0.5 b$ & $0.3 a$ & $0.5 b$ & 60.0 & - & - & - & - \\
\hline 10. & A-58 & $\times \mathrm{C}-141$ & & $0.3 \mathrm{a}$ & $0.5 \mathrm{c}$ & $0.4 b$ & 一 & 80.0 & $0.2 \mathrm{a}$ & $0.6 c$ & $0.3 b$ & 50.0 \\
\hline 11. & A-12 & $\times C-141$ & & - & $\longrightarrow$ & - & $\Longrightarrow$ & - & $0.6 \mathrm{~b}$ & $0.6 \mathrm{~b}$ & $0.4 \mathbf{a}$ & 66.7 \\
\hline 12. & A-125 & $\times C-181$ & & $0.3 \mathrm{a}$ & $0.4 b$ & $0.5 \mathrm{c}$ & $0.4 b$ & 125.0 & $0.3 \mathrm{a}$ & $0.4 b$ & $0.4 b$ & 100.0 \\
\hline 13. & A-181 & $\times C-181$ & & - & - & - & - & - & $0.4 \mathrm{a}$ & $0.4 a$ & $0.4 a$ & 100.0 \\
\hline 14. & A-16 & $\times \mathrm{C}-16$ & & $0.3 \mathrm{a}$ & $0.6 \mathrm{c}$ & $0.3 a$ & $0.4 b$ & 50.0 & $0.3 \mathrm{a}$ & $0.5 b$ & $0.3 a$ & 60.0 \\
\hline 15. & A-6 & $\times C-6$ & & $0.2 \mathrm{a}$ & $0.4 c$ & $0.3 b$ & $0.4 a$ & 75.0 & $\longrightarrow$ & - & - & - \\
\hline 16. & A-125 & $\times$ C-199 & & $0.3 a$ & $0.7 \mathrm{c}$ & $0.4 b$ & $0.4 b$ & 57.1 & - & - & - & - \\
\hline 17. & A-19 & $\times \mathrm{C}-19$ & & $0.5 \mathrm{c}$ & $0.4 b$ & $0.3 a$ & $0.4 b$ & 75.0 & - & - & - & - \\
\hline 18. & A-16 & $\times$ C-9 & & - & - & - & - & - & $0.3 a$ & $0.5 b$ & $0.3 \mathrm{a}$ & 60.0 \\
\hline 19. & A-101 & $\times$ C-9 & $\ldots \ldots$ & - & - & - & - & - & $0.4 \mathrm{a}$ & $05 b$ & $0.4 a$ & 80.0 \\
\hline 20. & A-181 & $\times C-9$ & & - & - & - & - & - & $0.4 b$ & $0.5 c$ & $0.3 a$ & 60.0 \\
\hline
\end{tabular}

Explanations as in Table 1. 
Table 6

Mean values $(x)$ and heterosis effect of onion bulb firmness (aacording to durometer readings)

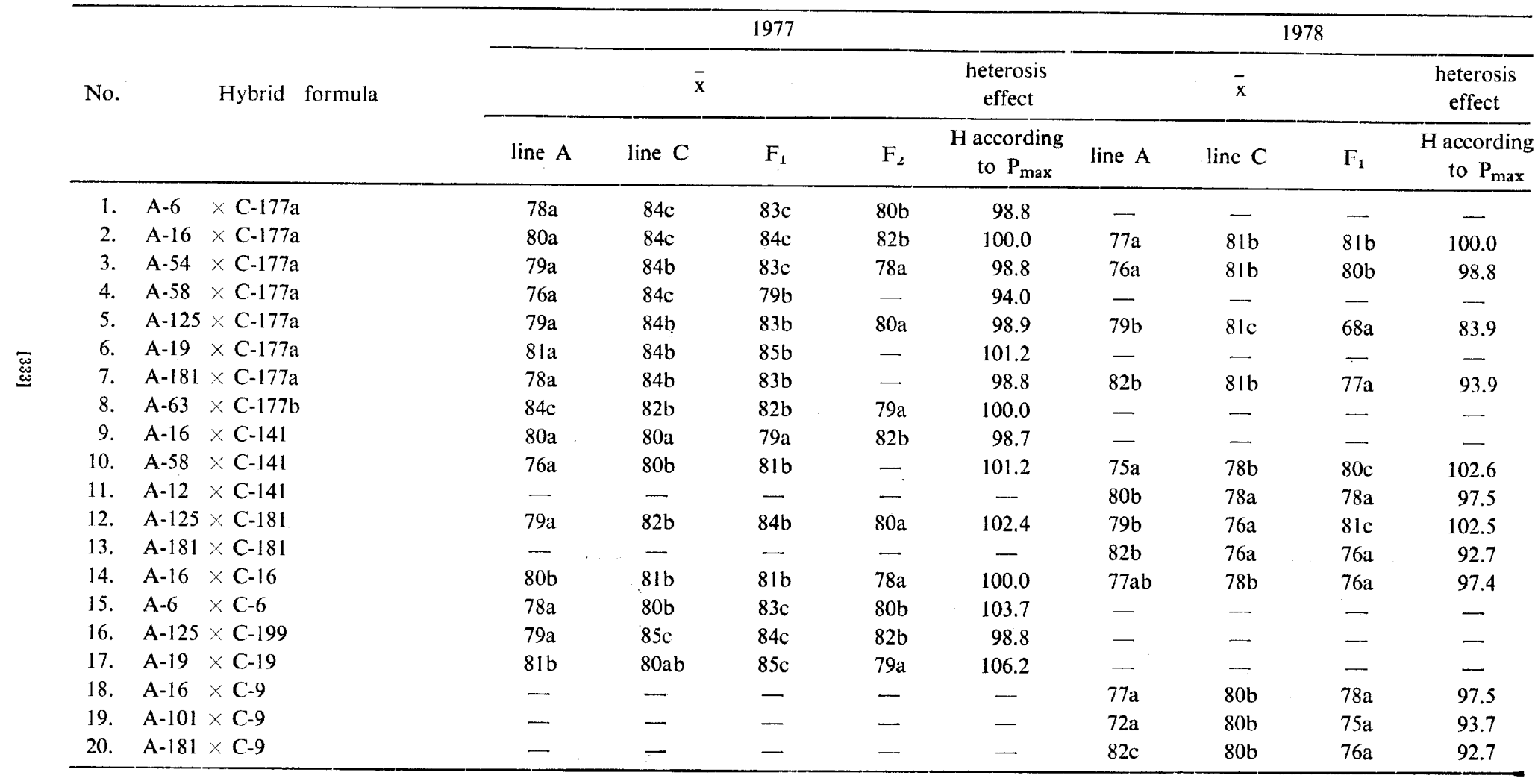

Explanations as in Table 1. 
T a b l e 7

Mean values $(\overline{\mathrm{x}})$ and heterosis effect of onion root disc diameter $(\mathrm{cm})$

\begin{tabular}{|c|c|c|c|c|c|c|c|c|c|c|c|}
\hline \multirow{3}{*}{ No. } & & \multirow{3}{*}{ Hybrid formula } & \multicolumn{5}{|c|}{1977} & \multicolumn{4}{|c|}{1978} \\
\hline & & & \multicolumn{4}{|c|}{$\bar{x}$} & \multirow{2}{*}{$\begin{array}{l}\begin{array}{c}\text { heterosis } \\
\text { effect }\end{array} \\
\text { according } \\
\text { to } P_{\max }\end{array}$} & \multicolumn{3}{|c|}{$\bar{x}$} & \multirow{2}{*}{$\begin{array}{c}\begin{array}{c}\text { heterosis } \\
\text { effect }\end{array} \\
\begin{array}{c}\text { H according } \\
\text { to } \mathbf{P}_{\text {max }}\end{array}\end{array}$} \\
\hline & & & line $A$ & line $\mathrm{C}$ & $F_{1}$ & $F_{2}$ & & line $\mathrm{A}$ & line $\mathrm{C}$ & $F_{1}$ & \\
\hline 1. & A- 6 & $\times C-177 a$ & $1.5 \mathrm{c}$ & $1.3 \mathrm{~b}$ & $1.3 \mathrm{~b}$ & $1.2 \mathrm{a}$ & 86.7 & - & - & - & - \\
\hline 2. & $A-16$ & $\times C-177 a$ & $1.4 \mathrm{c}$ & $1.3 \mathrm{~b}$ & $1.4 \mathrm{c}$ & $1.2 \mathrm{a}$ & 100.0 & $1.4 \mathrm{~b}$ & $1.2 \mathrm{a}$ & $1.4 \mathrm{~b}$ & 100.0 \\
\hline 3. & A-54 & $\times C-177 a$ & $1.5 \mathrm{c}$ & $1.3 \mathrm{~b}$ & $1.3 b$ & $1.1 \mathrm{a}$ & 86.7 & $1.3 \mathrm{~b}$ & $1.2 \mathrm{a}$ & $1.3 b$ & 100.0 \\
\hline 4. & A-58 & $\times C-177 a$ & $1.5 \mathrm{c}$ & $1.3 \mathrm{a}$ & $1.4 \mathrm{~b}$ & - & 93.3 & - & - & - & - \\
\hline 5. & A- 125 & $\times C-177 a$ & $1.4 \mathrm{c}$ & $1.3 b$ & $1.4 \mathrm{c}$ & $1.2 \mathrm{a}$ & 100.0 & $1.1 \mathrm{a}$ & $1.2 \mathrm{~b}$ & $1.1 \mathrm{a}$ & 91.7 \\
\hline 6. & A-19 & $\times C-177 \mathbf{a}$ & $1.4 \mathrm{~b}$ & $1.3 \mathrm{a}$ & $1.3 \mathrm{a}$ & - & 92.8 & - & - & - & - \\
\hline 7. & A-181 & $\times \mathrm{C}-177 \mathrm{a}$ & $1.4 b$ & $1.3 \mathrm{a}$ & $1.3 \mathrm{a}$ & - & 92.8 & $1.3 \mathrm{c}$ & $1.2 \mathrm{~b}$ & $1.1 \mathrm{a}$ & 84.6 \\
\hline 8. & A-63 & $\times \mathrm{C}-177 \mathrm{~b}$ & $1.4 b$ & $1.3 \mathrm{a}$ & $1.3 \mathrm{a}$ & $1.3 \mathrm{a}$ & 92.8 & - & - & $\cdots$ & - \\
\hline 9. & A-16 & $\times C-141$ & $1.4 \mathrm{c}$ & $1.2 \mathrm{a}$ & $1.3 \mathrm{~b}$ & $1.4 \mathrm{c}$ & 92.8 & - & - & - & - \\
\hline 10. & A-58 & $\times \mathrm{C}-141$ & $1.5 \mathrm{c}$ & $1.2 \mathrm{a}$ & $1.3 b$ & - & 86.7 & $1.3 \mathrm{c}$ & $1.1 \mathrm{a}$ & $1.2 \mathrm{~b}$ & 92.3 \\
\hline 11. & A-12 & $\times C-141$ & - & - & - & - & - & $1.1 \mathrm{a}$ & $1.1 \mathrm{a}$ & $1.3 \mathrm{~b}$ & 118.2 \\
\hline 12. & A- 125 & $\times$ C-181 & $1.4 \mathrm{c}$ & $1.3 \mathrm{~b}$ & $1.3 \mathrm{~b}$ & $1.2 \mathrm{a}$ & 92.8 & $1.1 \mathrm{a}$ & $1.2 \mathrm{~b}$ & $1.4 \mathrm{c}$ & 116.7 \\
\hline 13. & A-181 & $\times C-181$ & - & - & - & - & - & $1.3 \mathrm{~b}$ & $1.2 \mathrm{a}$ & $1.3 \mathrm{~b}$ & 100.0 \\
\hline 14. & A-16 & $\times \mathrm{C}-16$ & $1.4 \mathrm{a}$ & $1.4 a$ & $1.4 a$ & $1.4 \mathrm{a}$ & 100.0 & $1.4 c$ & $1.1 \mathrm{a}$ & $1.2 \mathrm{~b}$ & 85.7 \\
\hline 15. & A-6 & $\times$ C-6 & $1.5 \mathrm{c}$ & $1.2 \mathrm{a}$ & $1.4 b$ & $1.4 b$ & 93.3 & - & - & - & 一 \\
\hline 16. & A-125 & $\times$ C-199 & $1.4 b$ & $1.4 b$ & $1.3 \mathrm{a}$ & $1.4 \mathrm{~b}$ & 92.8 & - & - & - & - \\
\hline 17. & A-19 & $\times \mathrm{C}-19$ & $1.4 b$ & $1.3 \mathrm{a}$ & $1.3 \mathrm{a}$ & $1.3 \mathrm{a}$ & 92.8 & - & - & - & $一$ \\
\hline 18. & A-16 & × C-9 & - & - & - & $\ldots$ & - & $1.4 \mathrm{c}$ & $1.1 \mathrm{a}$ & $1.3 b$ & 92.8 \\
\hline 19. & A-101 & × C-9 & - & - & - & - & - & $1.3 b$ & $1.1 \mathrm{a}$ & $1.1 \mathrm{a}$ & 84.6 \\
\hline 20. & A-181 & $\times$ C -9 & - & - & - & - & - & $1.3 b$ & $1.1 \mathrm{a}$ & $1.3 b$ & 100.0 \\
\hline
\end{tabular}

Explanations as in Table 1. 
Table 8

Mean value $(\bar{x})$ and heterosis effect of dry matter and sugar contents of onion

\begin{tabular}{|c|c|c|c|c|c|c|c|c|c|c|c|c|c|c|c|c|c|c|}
\hline \multirow{4}{*}{ No. } & & \multirow{4}{*}{ Hybrid formula } & \multicolumn{12}{|c|}{ Mean value $(\bar{x})$} & \multicolumn{4}{|c|}{ Heterosis effect } \\
\hline & & & \multicolumn{4}{|c|}{ line $A$} & \multicolumn{4}{|c|}{ line $C$} & \multicolumn{4}{|c|}{$F_{1}$} & \multicolumn{2}{|c|}{ dry matter } & \multicolumn{2}{|c|}{ sugar } \\
\hline & & & \multicolumn{2}{|c|}{ dry matter } & \multicolumn{2}{|c|}{ sugar } & \multicolumn{2}{|c|}{ dry matter } & \multicolumn{2}{|c|}{ sugar } & \multicolumn{2}{|c|}{ dry matter } & \multicolumn{2}{|c|}{ sugar } & $\begin{array}{c}\text { by } \\
\text { dry- } \\
\text { ing }\end{array}$ & $\begin{array}{c}\text { by re- } \\
\text { frac- } \\
\text { tome- } \\
\text { ter }\end{array}$ & $\begin{array}{l}\text { mono- } \\
\text { sacch- } \\
\text { ari- } \\
\text { des }\end{array}$ & total \\
\hline & & & $\begin{array}{l}\stackrel{\infty}{\frac{\infty}{2}} \\
\frac{1}{0} \\
2\end{array}$ & 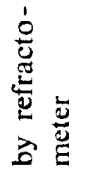 & 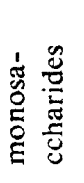 & $\stackrel{\bar{g}}{9}$ & 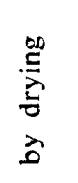 & 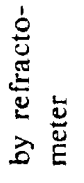 & 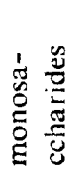 & $\bar{\Xi}$ & $\begin{array}{l}\frac{\infty}{5} \\
\frac{5}{5} \\
0 \\
0\end{array}$ & 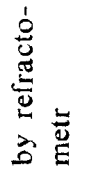 & 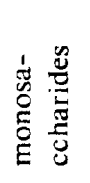 & $\bar{\sigma}$ & $\mathrm{H}$ & accordin & ng to & $P_{\max }$ \\
\hline 1. & A-6 & $\times C-177 a$ & 11.6 & 9.6 & 4.4 & 8.7 & 16.5 & 14.0 & 4.0 & 11.0 & 15.3 & 14.8 & 3.7 & 11.6 & 92.8 & 105.7 & 83.1 & 105.8 \\
\hline 2. & A-16 & $\times C-177 a$ & 11.6 & 10.2 & 5.1 & 8.1 & 16.5 & 14.0 & 4.0 & 11.0 & 13.6 & 13.0 & 4.3 & 10.7 & 82.6 & 92.8 & 84.2 & 97.6 \\
\hline 3. & A-54 & $\times C-177 a$ & 11.1 & 10.5 & 4.8 & 8.3 & 16.5 & 14.0 & 4.0 & 11.0 & 14.7 & 13.7 & 4.6 & 10.8 & 88.9 & 97.8 & 96.0 & 98.5 \\
\hline 4. & A-58 & $\times C-177 a$ & 11.6 & 10.9 & 4.7 & 8.7 & 16.5 & 14.0 & 4.0 & 11.0 & 13.8 & 12.1 & 42 & 9.2 & 83.9 & 86.4 & 90.2 & 83.3 \\
\hline 5. & A- 125 & $\times C-177 a$ & 12.4 & 10.9 & 4.5 & 8.5 & 16.5 & 14.0 & 4.0 & 11.0 & 14.4 & 13.0 & 4.1 & 10.1 & 87.3 & 92.8 & 91.9 & 91.9 \\
\hline 6. & A-19 & $\times C-177 a$ & 11.3 & 9.6 & 4.1 & 6.7 & 16.5 & 14.0 & 4.0 & 11.0 & 13.4 & 12.5 & 4.6 & 10.1 & 80.9 & 89.3 & 100.9 & 91.9 \\
\hline 7. & A- 181 & $\times C-177 a$ & 10.3 & 9.8 & 4.2 & 8.0 & 16.5 & 14.0 & 4.0 & 11.0 & 14.5 & 13.0 & 4.4 & 9.4 & 88.1 & 92.8 & 103.1 & 85.8 \\
\hline 8. & A- 63 & $\times C-177 b$ & 15.2 & 12.8 & 2.4 & 9.2 & 15.1 & 12.2 & 3.7 & 11.8 & 15.2 & 13.4 & 3.8 & 10.7 & 100.1 & 104.7 & 102.4 & 90.9 \\
\hline 9. & A-16 & $\times C-141$ & 11.6 & 10.2 & 5.1 & 8.1 & 13.2 & 11.4 & 3.6 & 8.9 & 12.1 & 11.0 & 4.3 & 8.8 & 91.6 & 96.5 & 84.8 & 98.7 \\
\hline 10. & A. 58 & $\times C=141$ & 11.6 & 10.9 & 4.7 & 8.7 & 132 & 11.4 & 3.6 & 8.9 & 11.9 & 10.7 & 3.6 & 8.2 & 90.3 & 93.8 & 76.2 & 92.3 \\
\hline 11. & A- 125 & $\times C-181$ & 12.4 & 10.9 & 4.5 & 8.5 & 11.0 & 9.8 & 3.7 & 7.1 & 11.4 & 10.9 & 3.8 & 7.0 & 92.5 & 100.0 & 84.5 & 82.3 \\
\hline 12. & A-16 & $\times C-16$ & 11.6 & 10.2 & 5.1 & 8.1 & 9.9 & 8.0 & 3.9 & 6.7 & 11.4 & 10.0 & 4.1 & 7.9 & 98.0 & 98.0 & 80.9 & 97.4 \\
\hline 13. & A-6 & $\times C-6$ & 11.6 & 9.6 & 4.4 & 8.7 & 14.5 & 12.9 & 3.7 & 10.0 & 14.5 & 14.0 & 3.6 & 11.0 & 100.5 & 108.5 & 79.0 & 110.5 \\
\hline 14. & A- 125 & $\times$ C-199 & 12.4 & 10.9 & 4.5 & 8.5 & 16.4 & 15.0 & 4.2 & 10.8 & 15.1 & 14.1 & 4.8 & 10.0 & 92.1 & 94.0 & 107.4 & 91.9 \\
\hline 15. & A. 19 & $\times C-19$ & 11.2 & 9.9 & 4.2 & 7.3 & 11.4 & 10.6 & 4.7 & 8.1 & 12.6 & 10.9 & 3.5 & 7.9 & 110.1 & 102.8 & 75.1 & 97.4 \\
\hline
\end{tabular}

Critical value of test $X_{2}:(a=0.05)=9.46$. 
results signalize the possibility of selecting individuals with better bulb firmness from the $F_{2}$ generation.

The root disc diameter was significantly smaller in $F_{1}$ hybrids (Table 7), which is a very desirable phenomenon from the point of view of bulb quality.

The average dry matter and sugar contents were highest in lines $\mathrm{C}-177 \mathrm{a}, \mathrm{C}-177 \mathrm{~b}$ and $\mathrm{C}-141(13-16 \%$ ) (Table 8). Whereas, in the remaining $\mathrm{A}$ and $\mathrm{C}$ lines, these contents were lower and amounted from 9 to $11 \%$. The dry matter and sugar contents in $F_{1}$ hybrids were intermediate between the parental forms. No evident heterosis was found, then, in most of the hybrids of the weight and sugar contents with the exception of $F_{2}$ hybrids $(A-19 \times C-19)$ and $(A-6 \times C-6)$. In addition to this, it was found that the parental lines and $F_{1}$ hybrids which were characterized by a high dry matter content also showed a higher sugar content.

\section{DISCUSSION}

One-half of the studied $F_{1}$ hybrids exhibited heterosis of bulb weight and diameter $(30-50 \%)$. These differences were significant at the $\alpha=0.05$ level. The large heterosis effect on these two traits with a high commercial significance also points to a high combining ability. This was not, however, studied in this experiment, because it had been the object of earlier investigations by this author (Dor uchow sk i, 1968).

In order to improve other bulb characteristics (dry skin thickness, bulb firmness, dry matter content) of $F_{1}$ hybrids, a large number of lines are necessary and parental components having the desired traits should be chosen.

The heterosis of such quantitative traits as bulb weight and diameter is undisputable proof for heterosis of quantitative traits. B r e w b a k r (1965) thinks that these types of problems are the subject of much interest of researchers engaged in quantitative and population genetics because this hypothesis is sometimes unduly disputed by some geneticists ( $\mathrm{K}$ u s m i e r, 1973).

The results obtained by this author in the study presented here are in agreement with the results of E 1-Shafie and A h med (1977), who believe that the heterosis of onion bulb weight may be high. The results of studies by $\mathrm{J}$ one s and $\mathrm{Clarke}(1943,1943-1947)$, J o nes and Davis (1944), Erickson and Gebelman (1954), Kobabe (1958) and Feltz (1975) have also shown that it is possible to obtain heterosis of weight. In $F_{1}$ hybrids, a higher percentage of bulbs with diameters exceeding $4 \mathrm{~cm}$ was found. The hybrids were characterized by good uniformity of bulb traits (e.g. size, shape, bulb color etc. K u- 
bik, 1975-1982), and some of them were well suited for mechanical harvest and sorting as well as for storage (S y p i en et al., 1978).

The weight of most of the $F_{2}$ hybrid bulbs fell significantly. These results support the widely held opinion that $F_{1}$ hybrids can only be used once, since heterosis and uniformity of bulb characteristics is found mainly in $F_{1}$. This is also indicated by the mean values and high variability of the remaining traits in $F_{2}$. The fall in the commercial value of hybrids was due to the segregation of characteristics in $F_{2}$. The onion bulbs of $F_{2}$ were very uneven in respect to almost all of the studied traits. The mean bulb weights in $F_{1}$ and $F_{2}(A-16 \times C-141)$ were very similar. No significant differences between them were found at the significance level of 0.05 . Segregation of traits in $F_{2}$ was not observed in that hybrid. It may seem as if this trait of $F_{1}$ was fixed in $F_{2}$ and passed down from generation to generation, against Mendel's laws. Most probably, the large number of cumulative genes by which the parental forms differed were responsible. That is why there was no segregation in $F_{2}$ because the intermediate forms in this generation were more numerous than the rare forms identical with the extremely differing parental forms. The individuals which surpass the parental forms in bulb weight, dry skin thickness, bulb firmness and dry matter may become the basis on which new varieties having better bulb characteristic than both of the parental forms can be developed.

The analysis of dry matter and sugar contents in the bulbs showed that analysis of dry matter by the drying method is more exact. The content of dry matter assayed this way was slightly higher than when it was determined refractometrically. However, the refractometric method is quicker and allows differences in the dry matter contents of the studied material to be determined quickly. It is also recommended by the IBPGR Allium (International Board for Plant Genetic Resources) (Astley et al., 1982) working within the framework of the FAO Genetic Resources Committee and by Nie uw h of et al. (1973).

Whole onions were used for analysis of their chemical composition in order to avoid mistakes in quantitative analysis; $\mathrm{N}$ i e u w h of et al. (1973) found that the dry matter content of the external, fleshy parts was lower than in whole onions.

\section{CONCLUSIONS}

1. Significantly high heterosis of bulb weight, diameter and uniformity of bulb characteristics in $F_{1}$ were found. Heterosis of dry skin thickness, bulb firmness and dry matter content was significant. 
2. The significant fall in bulb weight and worsening of uniformity of other traits in most of the $F_{2}$ generations confirmed the widely held view that $F_{1}$ hybrids should be used only once because trait segregation and lack of heterosis were found in $F_{2}$.

3. The lack of differences in mean bulb weights in $F_{1}$ and $F_{2}$ hybrids $(\mathrm{A}-16 \times \mathrm{C}-141)$ and significant surpassing of parental values indicates the probability that a large number of cumulative genes, by which the parental forms differed, were active.

4. The individuals surpassing their parental forms in bulb weight, dry skin thickness, bulb firmness and dry matter content in $F_{2}$ may constitute the basis of developing new varieties with better utilitarian traits than both of the parental forms used for the cross.

\section{REFERENCES}

Astley D., Innes N. L., van der Me r Q. P., 1982. Genetic resources of Allium species. Report of International Board for Plant Genetic Resources, pp. $1-38$.

A r un a ch a $1 \mathrm{~m} \mathrm{V.,} \mathrm{1977.} \mathrm{Heterosis} \mathrm{for} \mathrm{characters} \mathrm{governed} \mathrm{by} \mathrm{two} \mathrm{genes.} \mathrm{Journal}$ of Genetics 63: 15-24.

Brewbaker J. L., 1965. Agricultural Genetics. Prentice Hall Inc. New Yersey, USA, pp. 1-156.

Brzhnev D. D., 1971. Issledovanie belkov semen sortov i gibridov Allium cepa L. metodami ehlektroforeza i ikh spektroskopij. Fiziologiya Rast. 18: 206313.

Doruchowski R. W., 1968. Badania nad opracowaniem metody hodowli mieszańców heterozyjnych cebuli (Allium cepa L.) dla polskich warunków. Ph. D. thesis, Instytut Warzywnictwa, Skierniewice.

Doruchowski R. W., 1969. Dotychczasowe wyniki prac nad hodowlą mieszańców heterozyjnych cebuli. Biul. Warz. 9: 299-314.

Doruchowski R. W., 1971. Badania nad wartością kombinacyjną form rodzicielskich użytych do hodowli mieszańców heterozyjnych cebuli (Allium cepa L.) dla polskich warunków, Biul. Warz. 7: 131-145.

Doruchowski R. W., 1978. Polish onions: traditions, cultivars and $F_{1}$ hybrids. Eucarpia Meeting. Warsaw. October 5-10. 1976. Biul. Warz. 12: 19-25.

Doruchowski R. W., 1986. Variability and heritability of some onion (Allium cepa L.) characteristics of parental forms, $F_{1}$ hybrids and $F_{2}$ generation. Acta Agrobot. 39:

Dowker B. D., Fennell J. F. M., 1974. Some responses to agronomic treatments of different genotypes of bulb onions, Allium cepa L. J. Hort. Sci. 4: $1-14$.

E1-Shafie M. W., A hmed A. A., 1977. Inheritance of earliness and bulb weight in the common onion (Allium cepa L.). Libyan Journal of Agriculture 6: $253-266$.

Erickson H. T., Gabelman W. H., 1954. Potential value of inbreds and F: hybrid onions for seed production. Proc. Amer. Soc. Hort. Sci. 64: 393-398. 
Feltz H., 1975. Hybridzüchtung bei Zwiebelm. SAFA 27: 190.

J on es H. A., Clarke A. E., 1943-1947. History of hybrid onions. The Yearbook of Agriculture. Home Document 708. 79th: 320-326.

Jones H. A., Clarke A. E., 1943. Inheritance of male sterility in onion and the production of hybrid seed. Proc. Amer. Soc. Hort. Sci. 43: 189-194.

J ones H. A., Davis G. N., 1944. Inbreeding and heterosis and their relation to the development of new varieties of onions. USDA. Technical Bulletin 874: $1-28$.

K ob a be G., 1958. Entwicklugsgeschichtliche und genetische Untersuchungen an neuen männlich sterilen Mutanten der Küchenzwiebel (Allium cepa L.) Zeitung für Pflanzenzuchtung. 40: 352-384.

$\mathrm{Kubik}$ S., 1975-1982. Nieopublikowane sprawozdanie z prac wdrożeniowych. Warzywniczy Zakład Doświadczalny. Przyborów.

$\mathrm{Ku}$ ŝmier Ch. $\overline{\mathrm{F}}$., 1973. Genetic and physiological elements of heterosis. Sympozjum „Heterozja”. Warszawa 1971. Ossolineum.

Nieuwhof M., Bruyn J. W., De Sarretsen F., 1973. Methods to determine solidity and dry matter content of onions (Allium cepa L.). Fuphytica 22: $39-47$.

S y pień M., Kępk A., Kotlińska T., 1978. Evaluation of Polish $F_{1}$ hybrids of onion, as compared with standard cultivars of the Wolska type. Biul. Warz. 22: $31-34$.

Efekt heterozji niektórych cech użytkowych cebuli (Allium cepa L.)

\section{Streszczenie}

Badania na temat efektu heterozji niektórych cech użytkowych cebuli przeprowadzono w Zakładzie Hodowli i Genetyki Instytutu Warzywnictwa w Skierniewicach w latach 1977 i 1978. Przebadano 15 mieszańców $F_{1}, 11$ pokoleń $F_{2}$, 16 form rodzicielskich (8 linii męskosterylnych A i 8 linii wsobnych C).

Stwierdzono wysoki efekt heterozji masy, wysokości i średnicy, Heterozja twardości cebul, przylegania suchej luski i zawartości cukrów była nieznaczna Heterozja pozostałych cech (grubość szyjki, grubość piętki) byla niska. 